\title{
General relativistic results for a galactic disc in a multidimensional space-time
}

\author{
Carlos H. Coimbra-Araújo ${ }^{1}$ and Patricio S. Letelier ${ }^{2}$ \\ ${ }^{1}$ Instituto de Física Gleb Wataghin, Universidade Estadual de Campinas, CP 6165, \\ 13083-970 Campinas, SP, Brazil \\ ${ }^{2}$ Departamento de Matemática Aplicada, Instituto de Matemática, Estatística e Computação \\ Científica, Universidade Estadual de Campinas, 13083-970 Campinas, SP, Brazil
}

\begin{abstract}
We construct an exact and simple general relativistic model to describe a galactic disc based on a Schwarzschild disc immersed in a six dimensional space-time. The stability of this configuration is studied and we present results for the calculation of circular geodesic orbits.
\end{abstract}

Keywords. Gravitation - relativity - dark matter

\section{Introduction}

In general relativity, besides the solutions that currently offer important support to understand black holes and cosmology, actually the interest is extended for the case where the universe has more than four dimensions. Recently was proposed that a plausible reason for the gravitational force appear to be so weak can be its dilution in possibly existing extra-dimensions related to a bulk (the multidimensional space-time), where branes (the 4D space-time) are embedded (Dienes 1997; Lykken \& Randall 2000). In amendment, solutions of Einstein field equations for axially symmetric configurations have great astrophysical interest, because they can be used to model galaxies. In this sense, a long range of discs solutions was derived (follow for instance references in Vogt \& Letelier 2005). In the present work, as a first approach, we study the properties of a multidimensional axially symmetric matter configuration whose total density is located at the plane $z=0$ and is living in a $6 \mathrm{D}$ universe.

\section{Einstein equations and rotation curves for a static axially symmetric configuration with extra-dimensions}

The metric for an axial symmetric $6 \mathrm{D}$ space-time can be written in quasi-cylindrical coordinates as

$$
d s^{2}=-\mathrm{e}^{-\phi} \mathrm{d} t^{2}+f\left(\mathrm{~d} r^{2}+\mathrm{d} z^{2}\right)+h^{2} \mathrm{e}^{\phi} \mathrm{d} \varphi^{2}+\psi \mathrm{e}^{\nu} \mathrm{d} x^{2}+\mathrm{e}^{-\nu} \mathrm{d} y^{2},
$$

where $\phi=\phi(r, z), f=f(r, z), h=h(r, z), \psi=\psi(r, z)$ and $x$ and $y$ are the extradimensional coordinates. The vacuum Einstein equations $R_{A B}=0,(A, B=0,1, \ldots, 5)$ are obtained. We do $G=1$ and $c=1$.

To obtain a solution for Einstein equations which represents a thin disc located on $z=0$, we can introduce discontinuities in first derivatives of $f$ and $\phi$ by doing the replacement $z \rightarrow|z|+a$, where $a$ is a constant. In first approximation one can consider that the particles in the disc move along geodesics. By solving the integral of motion for the metric (2.1), we find the equation for rotation curves $V_{C}$ in function of specific extra-dimensional parameters. The Newtonian potential associated to a rod of constant density is used. In Fig. 1 we show the rotation curves for a disc in a extra-dimensional 
space-time, the stability of the model and in Fig. 2 the comparison to observational data and other models. The density profiles are the same as in the $4 \mathrm{D}$ case and we find that the extra-dimensions do not affect the density and the azimuthal and radial pressures.
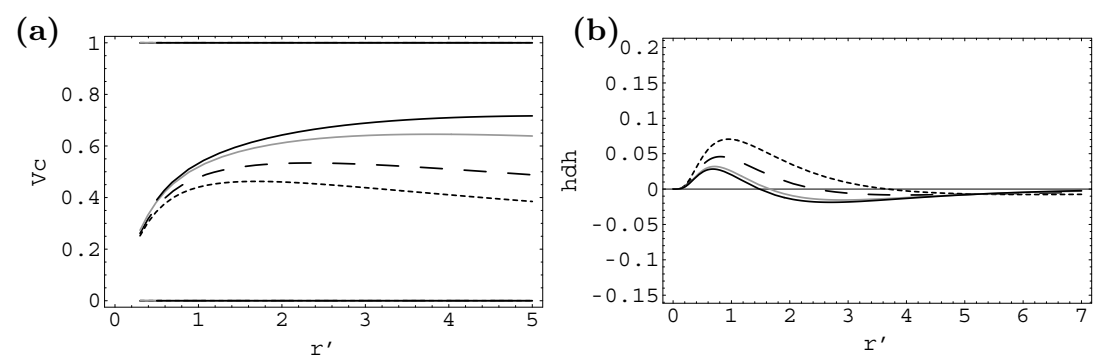

Figure 1. (a) Disc rotation curves with extra-dimensional parameters $C_{x}=0, C_{y}=0$, i.e., usual Newtonian profile (dotted line); $C_{x}=0.1, C_{y}=0.7$ (dashed line); $C_{x}=0.1, C_{y}=0.9$ (gray line); $C_{x}=0.1, C_{y}=0.95$ (full line). (b) Graphic showing the stability of the model. We take $r^{\prime}=r / m$ and the value used for $a$ is $a=1.5$ (the stability is reached when $a>1$ and when $h d h>0)$.

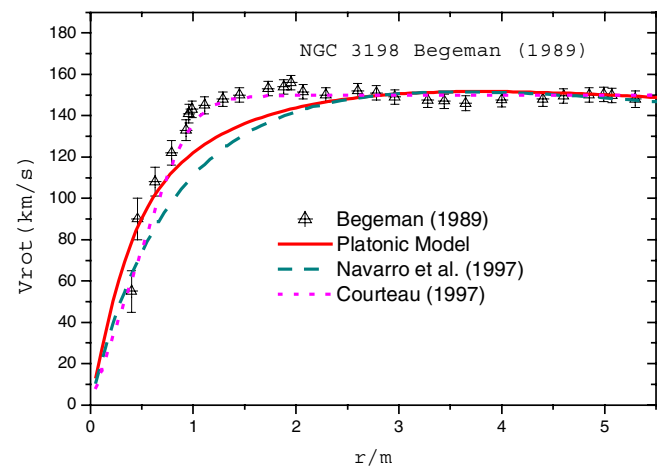

Figure 2. Different models - our model (Platonic), Navarro et al. (1997) and Courteau (1997) for the rotation curves of the NGC 3198 spiral galaxy. Observational data from Begeman (1989).

In this work we show with a simple example the construction of a disc embedded in a multidimensional space-time. The circular geodesic orbits of our model are stable in the region of interest and have the necessary accuracy to fit the rotation curves of many spiral galaxies.

\section{Acknowledgements}

C.H. C.-A. thanks CAPES and IAU for financial support and P.S.L thanks CNPq and FAPESP for partial financial support.

\section{References}

Begeman, K. G. 1989, A\&A, 223, 47

Courteau, S. 1997, Aj, 114, 2402

Dienes, K. R. 1997, Phys. Rep., 287, 447

Lykken, J. \& Randall, L. 2000, J. High En. Phys., 06, 014

Navarro, J. F., Frenk, C. S. \& White, S. D. M. 1997, Apj, 490, 493

Vogt, D. \& Letelier, P. S. 2005, mnras, 363, 268 